\title{
Book Review: Cosmogenic Radionuclides: Theory and Applications in the Terrestrial and Space Environments
}

\author{
P. Wilkinson \\ Bureau of Meteorology, IPS Radio and Space Services, P.O. Box 1386, Haymarket, NSW 1240, Australia
}

Correspondence to: P. Wilkinson (p.wilkinson@bom.gov.au)

Beer, J., Mccracken, K., and von Steiger, R.: Cosmogenic Radionuclides: Theory and Applications in the Terrestrial and Space Environments, Series: Physics of Earth and Space Environments, Springer-Verlag Berlin Heidelberg, Springer Heidelberg Dordrecht London New York, Library of Congress Control Number: 2011939486, 428 pp., doi:10.1007/978-3-642-14651-0, ISSN 1610-1677, eISSN 1865-0678, ISBN 978-3-642-14650-3, eISBN 978-3-64214651-0, € 139.99, 2012.

Since it was first formed, the Earth has been bathed in cosmic radiation generated by supernovae in our galaxy. Cosmic rays are accelerated to very high energies in supernova explosions and travel at almost the speed of light to the vicinity of our Sun. On arrival their intensity is modified as they pass through the heliosphere. On arrival near the Earth they are deflected by the geomagnetic field and collide with the Earth's atmosphere, producing unstable radioactive nuclides that are redistributed by terrestrial systems, including the atmosphere. Thus, generation of cosmogenic radionuclides is an important consequence of processes occurring in the galaxy, the Sun, and in the Earth's atmosphere. The half-lives of the cosmogenic radionuclides are so short compared to the age of the Earth that none would now exist on Earth without their continuous production through the interaction of cosmic rays with the atmosphere.

To read the history written in our environment by such cosmogenic nuclides, use has been made of a technique that has been evolving over the last few decades. Initially, there was no quantitative means of relating the variations in cosmogenic data to changes in the intensity of the cosmic radiation outside the magnetic field of the Earth. Subsequently, space observations provided the cosmic ray energy spectrum in interstellar space together with the manner in which solar activity influenced the spectrum at the Earth. This paved the way for the (in the authors' words) "majority of the material in this book and in the modern paleocosmic ray and cosmogenic nuclide literature".

The authors are well qualified in their respective fields: Jürg Beer is a pioneer in cosmogenic radionuclides measured in polar ice cores, Ken McCracken is a pioneer in cosmic ray research and space research from early satellites, and Rudolf von Steiger is director of the International Space Science Institute in Bern, whose research focusses on the solar wind. In their words, their aim is to (i) "address a wide audience, ranging from archaeology, biophysics, and geophysics, to atmospheric physics, hydrology, astrophysics and space science" and (ii) "to explain everything at the level of a graduate student without specialist skills in physics or mathematics". I feel they have succeeded admirably.

How about the content? What does it contain and does it deliver? The book is divided into four parts. After a brief useful introduction (Part 1), three roughly equally sized sections describe cosmogenic nuclides from their production to their applications. The first of these sections (Part 2 of the book) describes cosmic rays: their properties near the Earth, how these are measured and the time variations that are imposed on them. Cosmic rays have complex characteristics that vary from day to day, year to year and on into thousands of years - properties that complicate interpretation of the cosmogenic record. This knowledge is basic to what follows and this section provides a readily accessible background on cosmic rays. It concludes by summarising the properties of solar cosmic radiation.

Part 3 introduces cosmogenic nuclides, their production and how their transport and deposition are modelled. Modelling the cascade of particles produced when a highly energetic cosmic ray collides with the neutral atmosphere is achieved using mathematical simulation tools first developed by nuclear physicists. Some of the products of this chain of interactions are embedded in the Earth's surface; others are distributed within the environmental system. Understanding how the airborne products are created, distributed globally 
and finally deposited leads to the concept of how natural archives are formed and can be read. Successive chapters explain how the different nuclides are transported about in different geochemical cycles before they are deposited, forming chronological sequences that can be retrieved from ice and sediment cores and tree rings. This section concludes with a review of how nuclides are measured. Everything described up to this point in the book fails if the measurements cannot be carried out efficiently and accurately.

The last third of the book (Part 4) describes applications of nuclides to a range of applications: solar physics; galactic astronomy; the atmosphere; the hydrosphere; and the geosphere and biosphere. It concludes with a discussion of dating. The range of applications clearly demonstrates the scope of the techniques being developed.

To take just one of these applications as an example: as a space weather scientist I have followed with interest the publications leading up to the presentation of this book. Our understanding of the Sun's variations, from minutes to millennia, is an essential part of attributing risk factors and designing mitigation procedures in space weather impacts on technological systems. The gaps in this knowledge have been made clear by the unexpectedly low solar cycle 24 and the embedded super-storm that missed the Earth. How likely are super-storms; how likely are low solar cycles? Until we learn more about the inner functions of the Sun, we will have to rely on our knowledge of past solar observations, but the space-age observations are very brief and probably describe a special period of solar activity. In fact, the written history of man is negligible compared to the life of the Sun, which has forever beaten its message down on the Earth. How can we read this history? Can cosmogenic radionuclides answer all my questions? No - not yet. This is a developing field of research. To take one specific point: there appears to be clear evidence of major solar particle events in the cosmogenic archive. This offers important indications of the past impact of solar particle events at the Earth's surface, which leads to the prediction that low solar cycles can be even more hazardous than grand maximum cycles such as have been experienced during the space age. But this must be offset against the authors' warning that, as their book was being published (P119 footnote), recent ice cores did not corroborate some earlier observations of solar energetic particle events. On the other hand, there seems good evidence in the cosmogenic archive of many periods of low solar activity, possibly making solar cycle 24 more typical of the solar record than the last 50 years. I am confident that in the years ahead our knowledge of the past history of the Sun will be filled out as the cosmogenic archive is read more deeply and with increasing confidence - confidence based on the work presented in this book.
This book contains 196 figures (mostly coloured), 9 tables, 21 explanatory boxes, extensive reference lists at the end of each chapter, a complete list of abbreviations, a glossary and an effective index, all of which make the text readily accessible. The written text has been carefully prepared and as far as I could tell had no errors (either grammatical or typological) in it. I did not notice any obvious errors in the equations and, considering the care the authors have taken to explain these, I would be surprised if there were any. Summarising: the book is well written and the presentation is excellent. I look forward to further editions of this book when, I hope, the footnote on p. 119 will disappear, and I expect Part 4 will have to treble in size. 